\title{
Application of equivalent thermal circuits for calculation of thermal processes of induction cruel furnaces with a capacity of up to $1000 \mathrm{~kg}$
}

\author{
Abror Po'latov', Begali Mamadaliyev ${ }^{2}$, and Humoyun Mo'minov ${ }^{3}$ \\ ${ }^{1}$ Tashkent State Technical University, Department of Electromechanics and Electrotechnology, Universitetskaya - 2,Tashkent, 100095, \\ Uzbekistan \\ ${ }^{2}$ Tashkent State Technical University, Department of Electromechanics and Electrotechnology, Universitetskaya - 2,Tashkent, 100095, \\ Uzbekistan \\ ${ }^{3}$ Tashkent State Technical University, Department of Electrotechnica, Universitetskaya - 2,Tashkent, 100095, Uzbekistan
}

\begin{abstract}
The article describes new approaches to calculating the thermal regimes of induction crucible furnaces, as well as methods for calculating them using converted thermal circuits. The principles of constructing thermal models of structural parts and molten metal of induction crucible furnaces based on converted ETS and their use for the development of optimal systems for automatic control of the metal melting process are considered. The methodology for the development of ETS and the compilation of heat balance equations for induction crucible furnaces with a capacity of up to $1000 \mathrm{~kg}$ is presented.
\end{abstract}

Induction crucible furnaces used in foundries for smelting ferrous and non-ferrous metals have a large installed capacity. [nine]. The task of their control is to maintain the value of the maximum power during the period of melting and a given temperature during the holding period (to remove sulfur and impurities from the melt) before casting [4]. The problem of the synthesis of control systems for the thermal regime of the ITP is reduced to the development and hardware implementation of an algorithm that ensures the control of the melting mode of the metal, based on information about the average temperature of the metal being melted [5]. One of the most difficult problems in the design of ITP control systems is the development of a control system in transient modes of operation of this installation [4].

To study the heating processes of the main structural parts and the molten metal of the ITP using the ETS method, we select the number of thermal bodies, thermal conductivity between them and heat transfer to the environment.

The study of the thermal process is carried out on the basis of the following basic provisions and assumptions:

1. ITP is considered symmetric;

2. We consider that the values of thermal conductivity and heat transfer do not depend on temperature;

3. We believe that the electrical and thermal parameters are not distributed, but concentrated.

Taking into account the above conditions, an ITP with a capacity of up to $1000 \mathrm{~kg}$ with an open lid will compose an ETS with four thermal bodies: 1 - molten metal, 2 inductor, 3 - lining and 4 - case with internal heat sources (Fig. 1, a) [1] .
These thermal bodies are interconnected by the corresponding thermal conductivities, and the thermal bodies, the metal being melted and the body, also have heat transfer to the environment (Fig. 1, b), and each thermal body has, respectively, heat sources. For ETS, the system of equations for the heat balance of the steady and unsteady thermal regimes of the ITP is the basis for the mathematical models of these modes.

We compose a system of differential equations for the heat balance of the unsteady mode of operation of the ITP according to the presented ETS (Fig. 1, b):

$C_{1} \frac{d \Theta_{1}}{d t}+\Lambda_{11} \Theta_{1}-\Lambda_{12} \Theta_{2}-\Lambda_{13} \Theta_{3}=P_{1}$,

$C_{2} \frac{d \Theta_{2}}{d t}-\Lambda_{21} \Theta_{1}+\Lambda_{22} \Theta_{2}-\Lambda_{23} \Theta_{3}-\Delta_{24} \Theta_{4}=P_{2}$,

$C_{3} \frac{d \Theta_{3}}{d t}-\Lambda_{31} \Theta_{1}-\Lambda_{32} \Theta_{2}+\Lambda_{33} \Theta_{3}-\Lambda_{34} \Theta_{4}=P_{3}$,

$C_{4} \frac{d \Theta_{4}}{d t}-\Lambda_{42} \Theta_{2}-\Lambda_{43} \Theta_{3}+\Lambda_{44} \Theta_{4}=P_{4}$,

In the recording of the heat balance equations (1) and in the symbols in the ETS (Fig. 1, b),

the same designations are adopted - the indices mean the serial number of the active parts of the ITP under consideration;

- excess of the temperature of the molten metal, inductor, lining and housing of the ITP, $0 \mathrm{C}$;

- active and power losses of the corresponding parts of the ITP, $\mathrm{kW}$, in this case $\mathrm{P} 4=0$;

- thermal capacities of the corresponding parts of the ITP, $\mathrm{kW} \mathrm{s} / 0 \mathrm{C}$;

- thermal conductivity between the considered parts of the ITP; 
-total thermal conductivity of the considered parts of the ITP;

- heat transfer of the molten metal and the ITP body.

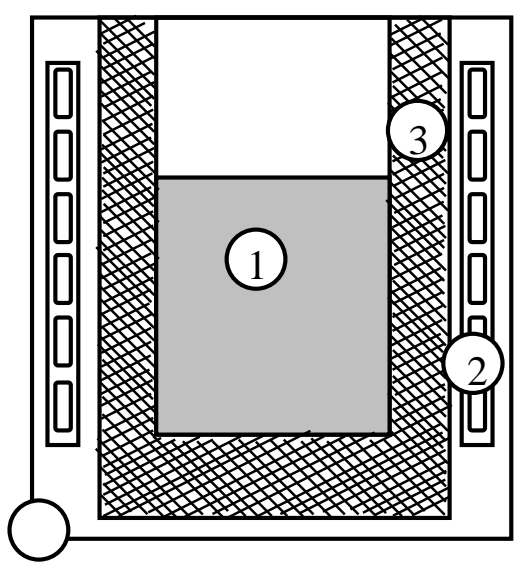

A)

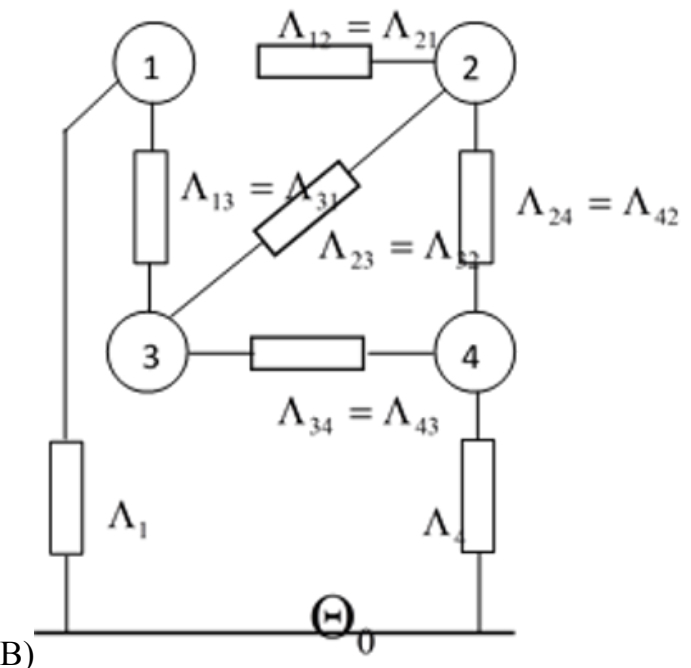

Fig. 1. Sketch (a) and the equivalent thermal circuit of the ITP with a capacity of up to $1000 \mathrm{~kg}$ (b)

The general view of the solution of this system of differential equations for heat balance (1) is as follows:

$$
\left.\begin{array}{l}
\Theta_{1}(t)=-A_{11} e^{-r_{1} t}-A_{12} e^{-r_{2} t}-A_{13} e^{-r_{3} t}-A_{14} e^{-r_{4} t}+\sum_{i=1}^{4} A_{1 i}, \\
\Theta_{2}(t)=-A_{21} e^{-r_{1} t}-A_{22} e^{-r_{2} t}-A_{23} e^{-r_{3} t}-A_{24} e^{-r_{4} t}+\sum_{i=1}^{4} A_{2 i}, \\
\Theta_{3}(t)=-A_{31} e^{-r_{1} t}-A_{32} e^{-r_{2} t}-A_{33} e^{-r_{3} t}-A_{34} e^{-r_{4} t}+\sum_{i=1}^{4} A_{3 i}, \\
\Theta_{4}(t)=-A_{41} e^{-r_{1} t}-A_{42} e^{-r_{2} t}-A_{43} e^{-r_{3} t}-A_{44} e^{-r_{4} t}+\sum_{i=1}^{4} A_{4 i}
\end{array}\right\}
$$

where the exponents of the roots of the characteristic equation (2.1), have the dimension s-1 and physically represent the values inverse to the heating time constants of the individual structural parts of the ITP and the molten metal; integration constants are determined from the initial and final conditions of integration; $I$ and $j$ are ordinal numbers of thermal bodies.

With the help of this calculation method, it is possible to calculate the unsteady heating process occurring in the molten metal and the main structural parts of the ITP and determine the temperature rise in the parts of the furnace of interest.
Since the steady-state heating mode of a crucible induction furnace is a special case of an unsteady system of equations (1), then, having adopted, we obtain a system of heat balance equations and write it in matrix form:

$$
\left[\begin{array}{l}
\Lambda_{11}-\Lambda_{12}-\Lambda_{13} 0 \\
-\Lambda_{21} \Lambda_{22}-\Lambda_{23}-\Lambda_{24} \\
-\Lambda_{31}-\Lambda_{32} \Lambda_{33}-\Lambda_{34} \\
0-\Lambda_{42}-\Lambda_{43} \Lambda_{44}
\end{array}\right] \times\left[\begin{array}{l}
\Theta_{1} \\
\Theta_{2} \\
\Theta_{3} \\
\Theta_{4}
\end{array}\right]=\left[\begin{array}{c}
P_{1} \\
P_{2} \\
P_{3} \\
P_{4}
\end{array}\right]
$$

The general view of the solution of the system of heat balance equations for the steady-state thermal process of the ITP for each considered thermal body has the following form:

$\Theta_{1}=\frac{\Delta_{11}}{\Delta} \mathrm{P}_{1}+\frac{\Delta_{12}}{\Delta} \mathrm{P}_{2}+\frac{\Delta_{13}}{\Delta} \mathrm{P}_{3}+\frac{\Delta_{14}}{\Delta} \mathrm{P}_{4}$,

$\Theta_{2}=\frac{\Delta_{21}}{\Delta} \mathrm{P}_{1}+\frac{\Delta_{22}}{\Delta} \mathrm{P}_{2}+\frac{\Delta_{33}}{\Delta} \mathrm{P}_{3}+\frac{\Delta_{24}}{\Delta} \mathrm{P}_{4}$,

$\Theta_{3}=\frac{\Delta_{31}}{\Delta} \mathrm{P}_{1}+\frac{\Delta_{32}}{\Delta} \mathrm{P}_{2}+\frac{\Delta_{33}}{\Delta} \mathrm{P}_{3}+\frac{\Delta_{34}}{\Delta} \mathrm{P}_{4}$,

$\Theta_{41}=\frac{\Delta_{41}}{\Delta} \mathrm{P}_{1}+\frac{\Delta_{42}}{\Delta} \mathrm{P}_{2}+\frac{\Delta_{43}}{\Delta} \mathrm{P}_{3}+\frac{\Delta_{44}}{\Delta} \mathrm{P}_{4}$

$$
\Delta=\mid \begin{aligned}
& \Lambda_{11}-\Lambda_{12}-\Lambda_{13} 0 \\
& -\Lambda_{21} \Lambda_{22}-\Lambda_{23}-\Lambda_{24} \\
& -\Lambda_{31}-\Lambda_{32} \Lambda_{33}-\Lambda_{34} \\
& 0-\Lambda_{42}-\Lambda_{43} \Lambda_{44}
\end{aligned}
$$

Where

determinant,

$$
\begin{aligned}
& \Delta_{11}=\left|\begin{array}{l}
\Lambda_{22}-\Lambda_{23}-\Lambda_{24} \\
-\Lambda_{32} \Lambda_{33}-\Lambda_{34} \\
-\Lambda_{42}-\Lambda_{43} \Lambda_{44}
\end{array}\right| \\
& \Delta_{12}=\left|\begin{array}{l}
-\Lambda_{21}-\Lambda_{23}-\Lambda_{24} \\
-\Lambda_{31} \Lambda_{33}-\Lambda_{34} \\
0-\Lambda_{43} \Lambda_{44}
\end{array}\right|, \quad \Delta_{13}=\left|\begin{array}{l}
-\Lambda_{21} \Lambda_{22}-\Lambda_{24} \\
-\Lambda_{31} \Lambda_{33}-\Lambda_{34} \\
0-\Lambda_{42} \Lambda_{44}
\end{array}\right| \text {, } \\
& \Delta_{14}=\left|\begin{array}{l}
-\Lambda_{21} \Lambda_{22}-\Lambda_{23} \\
-\Lambda_{31}-\Lambda_{32} \Lambda_{33} \\
0-\Lambda_{42}-\Lambda_{43}
\end{array}\right|, \quad \Delta_{21}=\left|\begin{array}{l}
-\Lambda_{12}-\Lambda_{13} 0 \\
-\Lambda_{32} \Lambda_{33}-\Lambda_{34} \\
-\Lambda_{42}-\Lambda_{43} \Lambda_{44}
\end{array}\right|, \\
& \Delta_{22}=\left|\begin{array}{l}
\Lambda_{11}-\Lambda_{13} 0 \\
-\Lambda_{31} \Lambda_{33}-\Lambda_{34} \\
0-\Lambda_{43} \Lambda_{44}
\end{array}\right| \\
& \Delta_{23}=\left|\begin{array}{l}
\Lambda_{11}-\Lambda_{12} 0 \\
-\Lambda_{31}-\Lambda_{32}-\Lambda_{34} \\
0-\Lambda_{42} \Lambda_{44}
\end{array}\right| \\
& \Delta_{24}=\left|\begin{array}{l}
\Lambda_{11}-\Lambda_{12}-\Lambda_{12} \\
-\Lambda_{31}-\Lambda_{32} \Lambda_{33} \\
0-\Lambda_{42}-\Lambda_{43}
\end{array}\right|, \quad \Delta_{31}=\left|\begin{array}{l}
-\Lambda_{12}-\Lambda_{13} 0 \\
\Lambda_{22}-\Lambda_{23}-\Lambda_{24} \\
-\Lambda_{42}-\Lambda_{43} \Lambda_{44}
\end{array}\right|,
\end{aligned}
$$




$$
\begin{gathered}
\Delta_{32}=\left|\begin{array}{l}
\Lambda_{11}-\Lambda_{13} 0 \\
-\Lambda_{21}-\Lambda_{23}-\Lambda_{24} \\
0-\Lambda_{43} \Lambda_{44}
\end{array}\right|, \Delta_{33}=\left|\begin{array}{l}
\Lambda_{11}-\Lambda_{12} 0 \\
-\Lambda_{21} \Lambda_{22}-\Lambda_{24} \\
0-\Lambda_{42} \Lambda_{44}
\end{array}\right| \\
\Delta_{34}=\left|\begin{array}{l}
\Lambda_{11}-\Lambda_{12}-\Lambda_{13} \\
-\Lambda_{21} \Lambda_{22}-\Lambda_{23} \\
0-\Lambda_{42}-\Lambda_{43}
\end{array}\right|, \quad \Delta_{41}=\left|\begin{array}{l}
-\Lambda_{12}-\Lambda_{13} 0 \\
\Lambda_{22}-\Lambda_{23}-\Lambda_{24} \\
-\Lambda_{32} \Lambda_{33}-\Lambda_{34}
\end{array}\right| \\
, \quad \Delta_{42}=\left|\begin{array}{l}
\Lambda_{11}-\Lambda_{13} 0 \\
-\Lambda_{21}-\Lambda_{23}-\Lambda_{24} \\
-\Lambda_{31} \Lambda_{33}-\Lambda_{34}
\end{array}\right|, \\
\Delta_{43}=\left|\begin{array}{l}
\Lambda_{11}-\Lambda_{12}-\Lambda_{13} \\
-\Lambda_{21} \Lambda_{22}-\Lambda_{13} 0 \\
-\Lambda_{31}-\Lambda_{21} \Lambda_{22}-\Lambda_{23}
\end{array}\right|, \quad \Lambda_{44}=\left|\begin{array}{l}
-\Lambda_{23} \\
-\Lambda_{31}-\Lambda_{32} \Lambda_{33}
\end{array}\right|
\end{gathered}
$$

private determinants.

The thermal conductivity, heat transfer, thermal capacities presented in the above equations are determined based on the specified geometric dimensions of the elements and parts of the crucible induction furnace and the thermophysical parameters of the materials used in them.

It should be noted that the calculation method presented above has a relatively complex algorithm, which complicates its widespread use in engineering practice. To facilitate the solution of the thermal problem as applied to engineering practice, further improvement of the thermal calculation method based on the converted equivalent thermal circuits is required.

\section{References}

1. Bessonov L.A. "Theoretical foundations of electrical engineering. Electrical circuits,"VSh, M., 1978.

2. Imamnazarov A.T. Use of signal-pole graphs for the study of thermal circuits. in the collection of scientific works of TashPI "Devices and Conversion Equipment," Tashkent, 1988, p.57-61.

3. Khashimov A.A., Imomnazarov A.T., Pulatov A.O. Mathematical model of metal melting processes in crucible furnaces. International Simposium on Helting by Electrotermic Sourses, Padua (Italy), June 22-25, 2004.

4. Khashimov A.A., Imamnazarov A.T., Pulatov A.A. Mathematical model of the metal smelting process in ICF based on linear graphs ./Collection of works of the 6th All-Russian Scientific and Technical Conference with international participation "Applied Aspects of Energy "//Russian Federation, Blagoveshchensk: ASU, 2011. 6 p. 5. Mathematical model of the metal melting process in ITP based on linear graphs. Collection of works. 6th AllRussian Scientific and Technical Conference. Applied aspects of energy. Russia. Blagoveshchensk-2011 Imamnazarov A.T. Pulatov A.A. Azamova G.A.
6. Khashimov A.A., Imamnazarov A.T., Pulatov A.A. Thermal modes of operation of induction crucible furnaces. - T .: Fan va texnologiya, 2013 .- 116b.

7. Pulatov Abror. Basic energy-saving principles at the enterprises of public catering. E3S Web of Conferences 216, 01136 (2020) RSES 2020. 